\title{
Politiques de la recherche environnementale et complexité du social ${ }^{\star}$
}

\author{
Bernard Ancori* \\ Épistémologie, Histoire des sciences et des techniques, Université de Strasbourg, IRIST, Strasbourg, France
}

Le type de recherche que nous avons à identifier dépend du type de finalité poursuivie par les politiques publiques, et la rationalité de ces dernières ne peut à son tour que s'appuyer sur des recherches finalisées qu'elles auront ainsi contribué à définir. Politique et recherche se nourrissent ainsi l'une l'autre en un cercle que nous souhaitons vertueux dans le cadre de nos sociétés éminemment complexes. Pour clarifier cette interaction, il me semble utile d'avoir recours à la théorie des systèmes adaptatifs complexes parce qu'elle a permis de renouveler un dilemme déjà ancien opposant deux stratégies possibles: une stratégie d'exploitation versus une stratégie d'exploration (encadré).

Je procéderai en trois points. Après un rapide rappel des difficultés propres à l'action publique confrontée à la transition écologique, j'exposerai le dilemme entre exploitation et exploration qui est au cœur du choix des politiques publiques visant à la réaliser et j'essaierai de montrer que le second terme de cette alternative est préférable au premier si le but politique visé est une transition écologique mise au service d'un développement durable. Je terminerai en proposant les recherches finalisées participatives comme une piste possible pour la mise en œuvre de cette stratégie d'exploration.

\section{Environnement et transition écologique: difficultés et complexité de l'action publique}

Concernant la question environnementale, l'action publique est contrainte d'emblée par une difficulté majeure qui tient au fait qu'elle se joue à différentes échelles de l'espace et du temps. Les acteurs publics ne maîtrisent pas l'espace, car l'enjeu environnemental

\footnotetext{
‡ Voir dans ce numéro, le texte d'introduction de Marcel Jollivet

* Auteur correspondant : ancori@unistra.fr
}

\begin{abstract}
Encadré. Le dilemme exploitation/exploration
Ce dilemme a été introduit dans le cadre de l'apprentissage organisationnel (Holland, 1975 ; March, 1991). L'exploitation consiste à raffiner et à étendre des compétences existantes dans le cadre de certitudes établies, et ses résultats sont positifs, à court terme et prévisibles. L'exploration consiste à expérimenter de nouvelles alternatives dont les résultats sont incertains, s'apprécient dans le long terme et se révèlent souvent négatifs. Les processus adaptatifs sont capables d'affiner plus rapidement l'exploitation que l'exploration, et rendent ainsi la première plus efficace à court terme que la seconde, mais la stratégie correspondante est potentiellement autodestructrice à long terme du fait de la perte de diversité des croyances des acteurs impliqués dans un processus d'apprentissage qui les mène inévitablement à converger ${ }^{1}$.
\end{abstract}

transcende toutes nos frontières administratives, sectorielles et disciplinaires (Lascoumes, 2012). Ils ne maîtrisent pas non plus le temps, car l'horizon temporel du politique se limite souvent au court terme des cycles électoraux, alors que l'incertitude radicale qui entoure la question environnementale est celle du temps long, voire très long.

Les difficultés tiennent également ici au fait que les processus de décisions se déroulent dans des arènes multiples et discontinues, où s'affrontent des acteurs ayant des points de vue différents, souvent contradictoires,

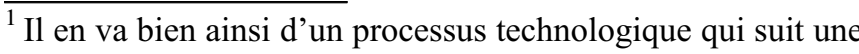
trajectoire donnée: des phénomènes de verrouillage (lock-in) apparaissent dans cette dernière dont le déploiement accroît toujours davantage la «dépendance par rapport au sentier» (path dependency) et rend simultanément de plus en plus difficile celui de trajectoires alternatives du fait du caractère exorbitant des coûts d'apprentissage impliqués au regard de ceux liés à la trajectoire initialement choisie (David, 1985).
} 
parfois conflictuels. Quant à la mise en œuvre des programmes d'actions ainsi décidées, elle reste souvent insatisfaisante, soit parce que le flou des objectifs laisse une grande marge de manœuvre aux acteurs locaux, soit parce que des objectifs précis sont rendus inatteignables du fait d'intérêts économiques - sans parler d'une pénurie chronique des moyens humains et financiers nécessaires pour mener ces programmes à bonne fin. Ces difficultés expliquent le caractère tardif de la prise en compte de la question environnementale par l'État français, et le fait que pendant longtemps les politiques correspondantes ont principalement été menées sur la base de revendications provenant d'interactions entre un milieu associatif relativement structuré et des réseaux administratifs et politiques sensibles aux enjeux environnementaux (Spanou, 1991 ; Lascoumes, 2012, p. 52 sq.).

Mais la question environnementale a encore gagné en complexité lorsque le rapport Brundtland a substitué en avril 1987 la notion de développement durable (ou soutenable) à celle d'environnement, car ce rapport sous-entendait ainsi que, précisément, le système de production en place n'était pas soutenable. Il liait de ce fait la question environnementale et la question sociale, qui apparurent dès lors comme étant les deux faces d'un seul et même problème - d'une seule et même crise, que certains lisent aujourd'hui comme une véritable crise de civilisation (Jollivet, 2015). D'où l'inscription de la question environnementale sur un agenda syndical attentif à sa dimension sociale, et la constitution d'une «nébuleuse verte » (Micoud, 2001) autour de l'ossature constituée par les associations environnementales. La myriade d'acteurs composant cette nébuleuse ajoute évidemment aux difficultés déjà évoquées de la prise de décision étatique, en la situant dans un cadre complexe. La complexité d'un système a été définie de multiples manières, mais toutes ces définitions disent que, toutes choses égales par ailleurs, elle est une fonction croissante du nombre de ses éléments et du nombre des interactions entre ces éléments, qu'ils soient naturels, sociaux ou économiques ${ }^{2}$.

Aux difficultés précédentes s'ajoute ainsi un délicat problème de coordination des acteurs impliqués par les

\footnotetext{
${ }^{2}$ Avec un collectif de $n$ acteurs, dont chacun peut communiquer avec chaque autre, il existe $n^{2}$ interactions possibles, et chacune d'entre elles pouvant exister (valeur 1) ou non (valeur 0 ), le nombre de structures de connexions possibles (c'est-àdire de combinaisons de ces $n^{2}$ interactions possibles) est alors au minimum égal à $2^{n 2}$. À chaque fois que l'on ajoute un acteur, on passe de $n^{2}$ à $(n+1)^{2}$ interactions possibles, donc d'un minimum de $2^{n 2}$ à $2^{(n+1) 2}$ structures de connexions possibles. Bref, le nombre de structures de connexions possibles augmente de manière exponentielle à chaque fois qu'un nouvel acteur rejoint la population de ceux qui existent déjà, et la complexité du système composé de ces acteurs et de ces structures s'en trouve accrue d'autant.
}

multiples enjeux de la transition écologique (Lascoumes, 2012). Nous sommes bien en présence d'un système complexe $\mathrm{e}^{3}$, et la question qui se pose en matière de développement durable centré sur cette transition est celle de la capacité d'adaptation de ce système. De manière générale, un système adaptatif complexe obtient de l'information à propos de son environnement et de ses interactions avec celui-ci, identifie des régularités au sein de cette information et condense ces régularités en formulant des modèles qui fondent son action dans le monde réel. Dans chaque cas, il existe plusieurs modèles en compétition, et l'action dans le monde réel exerce une influence rétroactive sur cette compétition, le but du système étant de sélectionner un modèle qui fonde un comportement adapté de sa part (Gell-Mann, 1995) - un comportement qui lui permette de persévérer dans son être. Projetons cette conception générale sur notre problématique propre: il s'agit ici d'identifier un comportement, issu des interactions entre politiques publiques et recherches finalisées, qui favoriserait la transition écologique orientée vers un développement durable au sens du rapport Brundtland, c'est-à-dire qui réponde aux besoins des générations présentes sans compromettre la capacité des générations futures de répondre aux leurs.

\section{Le dilemme exploration/exploitation}

De telles interactions s'inscrivent aujourd'hui dans un régime de production, de circulation et de certification des connaissances issu de la massification de l'enseignement supérieur (Gibbons et al., 1994 ; Nowotny et al., 2001). En effet, ce dernier a produit au niveau doctoral beaucoup plus de diplômés que le système académique ne pouvait en absorber. En dehors de celui-ci sont ainsi apparus de nouveaux gisements de producteurs potentiels de connaissances, et le développement de nouvelles technologies de l'information et de la communication a débouché sur une explosion de leurs connexions au début des années 1990. L'Université et les organismes de recherche ont ainsi perdu le monopole qu'ils pensaient avoir en matière de production des connaissances. En contraste avec un supposé régime de «tour d'ivoire» précédent, les connaissances sont désormais produites sur des bases transdisciplinaires par des consortiums de parties prenantes -académiques et non académiques - dans des contextes d'application, et elles s'inscrivent dans une perspective d'action. Ces connaissances circulent parmi toutes ces parties prenantes et sont évaluées par ces dernières, et ceci sur des critères aussi multiples et

\footnotetext{
${ }^{3}$ Pour une réflexion stimulante sur les liens entre complexité et environnement, voir Hess et Bourg (2016).
} 
variés que sont nombreux et divers les valeurs, les intérêts et les objectifs des acteurs concernés. L'organisation correspondante est ouverte, non hiérarchisée et réticulaire.

Ce régime de production, de circulation et de certification des connaissances supplante aujourd'hui nettement le précédent, de plus en plus repoussé aux marges du fonctionnement du monde académique. Le modèle d'expertise s'est transformé en conséquence: auparavant fondée sur une science neutre et capable de distinguer clairement entre faits et valeurs dans un monde de risques probabilisables, l'expertise scientifique est devenue aujourd'hui une expertise sociotechnique fondée sur une techno-science non neutre où faits et valeurs ne cessent de s'interpénétrer dans un monde d'incertitude radicale (Pestre, 2006, 2010). L'une de ces valeurs tend cependant de plus en plus à supplanter toutes les autres, car aujourd'hui les sciences et les technologies sont largement soumises à une contrainte de rentabilité économique (Pestre, 2003, 2013). Sous cette contrainte qui simplifie le problème de la coordination entre les acteurs en inscrivant la décision dans le seul cadre de l'analyse coûts/bénéfices, elles sont pilotées politiquement dans un souci de compétitivité, suivant en cela l'objectif hautement affiché par la déclaration de Lisbonne de mars 2000, et les impératifs de l'économie s'opposent le plus souvent à ceux d'un développement durable.

Le dilemme entre stratégie d'exploration et stratégie d'exploitation montre cependant qu'une politique favorisant des recherches orientées vers des objectifs principalement conçus à court terme dans un cadre étroitement économique sur fond de concurrence internationale n'est guère adaptée à la réalisation d'une transition écologique et un développement durable dont les enjeux concernent à court et à long terme la planète entière.

En généralisant le dilemme entre exploitation et exploration à l'ensemble des systèmes adaptatifs complexes, la recherche a montré que l'équilibre entre exploitation et exploration était pertinent dans toute une gamme de situations. Il existe cependant quatre grands types de situations dont chacun devrait conduire rationnellement à préférer l'exploration à l'exploitation (Axelrod et Cohen, 1999, pp. 89-111) (encadré).

Il ne fait aucun doute que la question environnementale se pose dans une situation du premier type, car c'est bien là une question à long terme, et elle se situe à un niveau suffisamment général pour que la gamme des applications possibles de ses solutions soit très étendue. Il est également hautement probable que cette question relève du quatrième type de situations, car la poursuite de la stratégie d'exploitation actuelle semble devoir conduire l'humanité à un désastre: en témoignent

\section{Encadré. Les types de situations dans lesquelles il est} préférable de se situer dans une optique d'exploration

Le premier type de telles situations est celui de problèmes à long terme ou très vastes, car plus il est possible d'utiliser dans le temps ou dans l'espace une amélioration due à une stratégie d'exploration, plus il est avantageux de supporter le coût de sa recherche.

Le deuxième type de situations est celui de problèmes dont les solutions possibles ont un impact rapide et fiable. En effet, dans ce cas l'on peut s'apercevoir rapidement qu'une solution inédite est meilleure qu'une solution éprouvée, et ceci avec peu de risques d'erreurs. Quand ces conditions d'impact rapide et fiable des solutions n'existent pas, il vaut souvent la peine d'essayer de les créer afin de tirer bénéfice de l'exploration.

Le troisième type de situations en faveur de cette dernière est celui de problèmes pour lesquels elle a peu de chances d'occasionner des catastrophes. Le risque d'un résultat d'exploration très mauvais est alors très faible, et ceci d'autant plus qu'il est possible de multiplier les mêmes expériences, de sorte que le système global ne se trouve pas à la merci de l'échec d'une seule, tout en pouvant escompter la réussite de plusieurs.

Enfin, le quatrième type de situations militant pour l'exploration est celui où la poursuite de l'exploitation de la meilleure solution connue à ce jour semble devoir conduire à un désastre. L'attrait de l'exploration vient ici des conséquences potentiellement négatives de l'exploitation.

les prédictions du rapport Meadows émises en $1972^{4}$ (déclin des ressources non renouvelables restantes ou augmentation des niveaux de pollution), qui se virent remarquablement confirmées par les tendances observées en l'an 2000. Et sur la base du prolongement de ces tendances, le principal auteur de ce rapport a récemment réitéré (Le Monde, 23 mai 2012) son annonce d'une situation d'effondrement de l'économie et de déclin de la population pour 2030 si les décideurs politiques ne prennent pas les mesures nécessaires au maintien d'un «état d'équilibre global»-s'ils persistent dans leur stratégie d'exploitation.

Telles sont les deux premières raisons, l'une positive et l'autre négative, dont chacune devrait inciter des politiques publiques visant à réaliser la transition écologique à privilégier une stratégie d'exploration par rapport à la stratégie actuelle d'exploitation. Nous allons voir qu'il en va de même pour chacune des deux autres types de situations, en présentant une piste possible en matière de recherches finalisées adaptées à une telle stratégie.

\footnotetext{
$\overline{4}$ Meadows et al. (1972).
} 


\section{Une piste possible: la recherche participative sous forme de forums hybrides}

Il existe différentes manières de réduire la complexité d'un système, qui se ramènent toutes à partitionner nos ensembles d'acteurs et de structures de connexions en le moins de sous-ensembles que possible sur la base de leurs ressemblances (Simon, 1962). À la limite, si tous ces acteurs et structures étaient strictement semblables, leur ensemble pourrait ainsi être réduit à un et un seul acteur en interaction avec lui-même; et à l'inverse, si aucun acteur ni aucune structure de connexion ne ressemblaient en rien à aucun ni à aucune autre, la complexité de leur système serait irréductible.

C'est la nécessité de réduire cette complexité qui conduit une organisation à se doter d'un porte-parole censé représenter ses membres auprès d'un tiers. Sur le plan politique et institutionnel, c'est là une raison technique évidente de préférer la démocratie représentative à la démocratie directe. Encore faut-il que cette démocratie représentative tienne ses promesses, et se donne pour visée la réalisation d'un bien commun à tous les acteurs qui acceptent de déléguer leurs pouvoirs et d'être ainsi représentés. Or, le problème de coordination évoqué plus haut semble aujourd'hui rendre cette réalisation impossible en matière de transition écologique et de développement durable. Nous pouvons d'ailleurs penser que c'est précisément pour tenter de résoudre ce problème en sélectionnant leurs interlocuteurs afin de réduire leur diversité, et donc la complexité de l'ensemble du système, que les pouvoirs publics nationaux et européens ont conféré un tel privilège à la dimension économique des recherches scientifiques et technologiques. Ainsi interprétée dans le cadre d'une stratégie d'exploitation ${ }^{5}$, leur

\footnotetext{
${ }^{5}$ Une telle stratégie reposerait ainsi sur un double préjugé. Le premier est le préjugé technophile, conscient et explicite, qui considère que quels que soient les effets négatifs d'un état donné du développement technologique, un état ultérieur permettra de les corriger, voire de les annihiler-d'où le privilège donné à des mesures a posteriori, dénoncé par le rapport Brundtland. Le second est implicite, voire inconscient, et il repose sur le vieux présupposé libéral introduit au XVIII ${ }^{\mathrm{e}}$ siècle par Adam Smith sous le concept de «main invisible». Avec ce concept, il s'agissait alors pour le fondateur de l'économie politique de justifier le primat donné à la production de biens et services sur leur répartition: la croissance ainsi obtenue devait ensuite tout naturellement conduire à une répartition de ses fruits parmi la population, à la fois juste et propre à assurer à chacun un niveau de vie supérieur à celui qu'il obtiendrait si l'État commettait l'erreur de penser, en inversant à tort les termes de la causalité, que la répartition des biens et services contribuait à déterminer le niveau de la production au lieu d'en dépendre.
}

action a certes réduit la complexité du système des acteurs et de leurs structures de connexions possibles, mais elle n'a certainement pas contribué à rendre ce système adaptatif - bien au contraire. Sur la base du premier et du quatrième type de situations appelant une stratégie d'exploration, l'autre stratégie se révèle donc inadaptée à la question environnementale.

Il en va de même pour le deuxième type de situations : celui de problèmes dont les solutions possibles ont un impact rapide et fiable, de sorte que l'on peut vite s'apercevoir, avec une faible marge d'erreur, qu'une solution inédite est meilleure qu'une solution éprouvée. Nous avons également relevé qu'il valait souvent la peine d'essayer de créer ce type de situations quand il n'existait pas. Or, c'est très précisément ce type de situations qu'une politique de recherche orientée vers la transition écologique pourrait créer aujourd'hui en soutenant des recherches coopératives sur la question environnementale.

Pour concrétiser les stratégies d'exploration, il existe de multiples modalités de recherches dites «participatives» (Houllier, 2016). Dans leur acception la plus radicale, de telles recherches placent savants et profanes sur un pied d'égalité tout au long du processus de recherche: depuis son extrême amont (définition de l'objet de la recherche, agenda et méthodologie) jusqu'à son extrême aval (livraison et évaluation du produit de la recherche), ce processus est construit et piloté conjointement par l'ensemble non hiérarchisé de ses parties prenantes. Cette égalité de principe repose sur une condition nécessaire dont la réalisation consiste en deux changements simultanés de postures épistémologiques de la part des acteurs concernés. Elle suppose d'abord que les «savants » prennent conscience des séries de pluralité-disciplinaire, stylistique, méthodologique, axiologique, temporelle - du travail scientifique et conçoivent sur cette base une science au pluriel et une épistémologie des sciences impliquées (Coutellec, 2015), et qu'ils poussent leur épistémologie impliquée au-delà de leur seul monde académique. Abandonner l'illusion répandue selon laquelle ce monde occuperait une position de surplomb qui donnerait à son point de vue un caractère transcendant, et en concevoir en conséquence ses connaissances comme une partie prenante parmi d'autres de chaque situation problématique, sans prétendre au moindre privilège quant à la manière dont le problème est formulé et dont ses solutions sont envisagées (Alix et al., 2008 ; Pestre, 2008): tel est le premier changement de posture épistémologique nécessaire à la réalisation d'une forme radicale de recherches participatives.

Quant aux «profanes », que leur forme d'engagement soit celle d'experts-militants ou d'êtres vivants sensibles (Micoud, 2007), ils ont à parcourir en sens inverse le 
même chemin que les savants, en répondant en miroir à l'humilité nouvelle de ces derniers par l'abandon de leur humilité propre. Car cette dernière provient identiquement de la vision d'un savoir qui serait organisé en une hiérarchie unique au sommet de laquelle trôneraient des connaissances scientifiques dominant de toute leur hauteur leurs savoirs "de terrain » ou "expérientiels », toujours localement englués dans une gangue d'empiricité qui leur interdirait toute possibilité de montée en généralité. Une telle vision obère l'indispensable participation active des profanes ${ }^{6}$ à la réalisation d'une transition écologique mise au service d'un développement durable, alors même que cette position scientiste apparaît aujourd'hui épistémologiquement périmée (Calame, 2011 ; Lipinsky, 2011 ; Stengers, 2013). Son abandon par ces mêmes " profanes » témoignerait d'un changement de posture épistémologique rigoureusement symétrique de celui des "savants », et contribuerait tout autant à rendre possible la réalisation de recherches participatives telles que définies ci-dessus.

S'inscrivant dans le sillage d'une longue revendication de démocratie participative (Bacqué et Sintomer, 2011), qui s'exprime notamment dans le domaine des sciences et des technologies (Ancori, 2012 ; Bonneuil et Joly, 2013 ; Callon et al., 2001 ; Pestre, 2011), cette condition nécessaire à la promotion de recherches participatives s'accompagne d'une condition suffisante. Celle-ci consiste en une volonté politique des autorités étatiques de favoriser ce type de recherches finalisées qui apparaît aujourd'hui le plus apte à leur offrir les connaissances et les savoirs permettant d'asseoir leurs décisions sur une base collectivement et rationnellement construite, et de les rendre ainsi socialement acceptables. Ces autorités pourraient notamment privilégier la tenue de «forums hybrides», car l'objet de tels forums est souvent issu de préoccupations locales, et ils débouchent généralement sur des solutions fiables du fait d'être construites conjointement par l'ensemble des parties prenantes (Callon et al., 2001). Cela va dans le sens des travaux d'Ostrom (1990), qui montrent que les biens communs peuvent être efficacement gérés par les acteurs les plus directement concernés, d'où le privilège qu'elle accorde aux stratégies les plus territorialisées possibles. Elle identifie huit principes caractéristiques des collecti-

\footnotetext{
${ }^{6}$ Le principe de cette participation active était déjà affirmé dans le discours que le président Georges Pompidou prononça le 28 février 1970 lors de son voyage aux États-Unis : dans la «morale de l'environnement » qu'il appelait alors de ses vœux, s'imposait «plus qu'ailleurs le contrôle des citoyens et leur participation effective à l'aménagement du cadre de leur existence.», cité par Laville (2010).
}

vités robustes de gestion de ressources communes, qui échappent ainsi au tout-marché comme au tout-État. Parmi les facteurs favorisant la coopération entre acteurs ayant des intérêts divergents, et souvent conflictuels, les principaux sont la prise de conscience collective des enjeux à long terme et la confiance fondée sur un partage équitable des contraintes. De fait, comme le note Lascoumes (2012), «l'obligation de concertation avec les populations locales est devenue un passage obligé pour la plupart des projets d'aménagement locaux ; mais aussi de grande ampleur » (p. 37). Ainsi, la conférence de Rio de 2012 a notamment débouché sur l'énoncé de 27 principes, dont le $\mathrm{n}^{\mathrm{o}} 10$ stipule que les programmes de développement durable «doivent reposer sur une participation des populations concernées à la décision et à la gestion. » (Lascoumes, 2012, p. 43). Il en va de même pour ce bien commun que représentent l'Internet et les documents numériques, aujourd'hui soumis à la même tentative d'enclosure que les «communs » anglais que se disputèrent les pauvres des campagnes et les propriétaires terriens entre le XIII ${ }^{\mathrm{e}}$ et le XVII ${ }^{\mathrm{e}}$ siècle (Le Crosnier, 2012) ${ }^{7}$. À condition de laisser à ce type de forums le temps long qui est nécessaire pour identifier, tester et valider ces solutions, une telle stratégie d'exploration résoudrait le problème de la coordination des acteurs en leur permettant de s'auto-organiser, et elle serait davantage de nature à favoriser la transition écologique souhaitée qu'une stratégie d'exploitation mettant souvent écologie et économie en opposition et privilégiant systématiquement cette dernière.

D'ores et déjà, trois des quatre situations énoncées plus haut incitent à opter pour une stratégie d'exploration. Il nous reste à étudier le troisième type de situations militant en ce sens : celui de problèmes pour lesquels une telle stratégie a peu de chances d'occasionner des catastrophes, et ceci d'autant moins qu'il est possible de multiplier les mêmes expériences et de prémunir ainsi le système global contre l'échec d'une seule qui viendrait confirmer la tendance d'une telle stratégie à mener à des résultats négatifs. Or, en matière d'environnement tel est à l'évidence le cas des recherches participatives, tant la diversité du monde associatif en ce domaine et la variété des problématiques locales concernées autorisent la multiplication des expériences en territoire. Certes, ce n'est ainsi jamais exactement la même expérience qui est tentée, mais cette diversité peut néanmoins être ramenée

\footnotetext{
${ }^{7}$ Pour un argumentaire très intéressant en faveur d'un « droit du commun » comme principe politique de la co-activité ou de la co-participation, voir Laval et Dardot (2015). Sur une recension des actions «collaboratives» menées aujourd'hui sur ce thème, voir Berlingen (2015).
} 
à quelques profils-types idéaux ${ }^{8}$ dont il est possible de tirer quelques enseignements de portée générale, voire des règles de «bonnes pratiques».

La montée en généralité permise par ces enseignements et ces bonnes pratiques serait grandement favorisée par la fédération en réseaux - voire en un réseau unique - de ces initiatives aujourd'hui dispersées. L'émergence de tels réseaux aurait un double avantage. Sur le plan des politiques de recherche, installant ces réseaux (ou ce réseau) en tant que « tiers » entre collectifs de recherche participative et pouvoirs publics, elle réduirait significativement la diversité des acteurs et de leurs structures de connexions possibles aux yeux de ces derniers. Ce faisant, elle diminuerait la complexité du système auquel ils sont confrontés - et cette fois-ci, de manière à rendre ce système complexe réellement adaptatif. Et au niveau des recherches finalisées, elle contribuerait à favoriser l'apparition d'une slow science face à la science « dure » dont le mode de fonctionnement domine aujourd'hui une orientation technico-marchande héritée du XIX ${ }^{\mathrm{e}}$ siècle (Stengers, 2013). En effet, il s'agit là de deux conventions concurrentes au sens de Lewis $(1969,1983)$, et la domination actuelle de la seconde sur la première ne pourrait prendre fin que si cette slow science pouvait s'appuyer sur des réseaux localisés s'organisant progressivement en un front unique tissant des liens étroits entre un nombre suffisamment élevé de ses composants (Ancori, 2017 ; Boyer et Orléan, 1994).

Si tel était le cas, cette rupture de cadre dans les représentations des acteurs serait en outre de nature à accroître la créativité et la productivité de l'activité scientifique et technique par rapport à la situation actuelle où elles semblent s'essouffler (Larivière et al., 2008). En effet, la diversité des croyances des acteurs d'une slow science conjuguant les apports des « savants » et des «profanes» serait bien plus grande que celle de la science «dure» actuelle, et à condition de ne pas rendre impossible la communication entre les acteurs, cette diversité serait source d'un surcroît de créativité

\footnotetext{
${ }^{8} C f$. la synthèse du Colloque Repère des 15 et 16 mai 2014, qui réduit la diversité des 18 projets soutenus entre 2010 et 2014 à trois profils-types. Le premier vise une meilleure interaction entre chercheurs et associations, et à contribuer à une mutation de la démocratie vers une forme dialogique. Le deuxième rassemble les différentes parties prenantes d'une controverse, et peut voir coexister deux types de travail critique - radical ou régulateur. Le troisième converge explicitement autour de valeurs partagées et vise à construire une «coalition de cause » où le travail critique est plutôt de type régulateur. (http://www. programme-repere.fr/)
}

potentielle (Ancori, 2014)9. Et s'il est vrai que le principal moteur de nos économies actuelles consiste en le développement des sciences et des technologies, ce serait finalement ces économies elles-mêmes qui pourraient en bénéficier. Écologie pourrait ainsi rimer avec économie, au lieu de s'opposer l'une à l'autre comme c'est souvent aujourd'hui le cas.

En définitive, la stratégie d'exploration, ainsi amplement justifiée et recommandée en vue de réaliser la transition écologique, consisterait tout simplement à pousser d'un cran le mouvement amorcé par le passage à un mode de production, de circulation et de certification des connaissances dans lequel l'université et les organismes de recherche ont perdu leur monopole. Au lieu de se contenter de partager ce dernier avec une population certes élargie d'acteurs, mais qui ne constitue aujourd'hui qu'une faible partie de la société globale, il s'agirait de prolonger et d'élargir encore ce passage en impliquant l'ensemble de la population d'acteurs concernés par la question environnementale. En reconnaissant chacun de ces derniers comme détenteur d'un savoir singulier, et la qualité de l'apport potentiel de ce savoir à un processus collectif de recherche dont toutes les parties prenantes seraient également légitimes, elle élargirait le mouvement vers les sciences impliquées souhaitées par Coutellec (2015), permettant ainsi d'enrôler tous les acteurs au service du bien commun. À la faveur des «sociétés de la connaissance» qui remplaceraient ainsi les «économies de la connaissance » actuelles, le Politique reprendrait la main sur un paysage d'enseignement supérieur et de recherche largement livré aujourd'hui au jeu des intérêts économiques et financiers. Saura-t-il et voudra-t-il saisir le kairos - ce moment opportun pour l'action - pour ce faire?

\section{Références}

Alix J.-P., Ancori B., Petit P. (Eds.), 2008. Sciences en société au XXI siècle: autres relations, autres pratiques, Paris, CNRS Editions.

Ancori B., 2012. La production et la circulation des connaissances scientifiques et des savoirs profanes dans nos sociétés techniciennes, in Darbellay F. (Ed.), La circulation des savoirs. Interdisciplinarité, concepts nomades, analogies, métaphores, Berne, Peter Lang, 203-240.

\footnotetext{
${ }^{9}$ C'est l'une des explications de la nécessité d'un temps «long» des recherches participatives, souvent mentionnée dans les analyses de ce domaine: source de créativité potentielle, la diversité des acteurs est également un frein à l'établissement d'un langage commun entre ces derniers, de sorte que le temps «long» évoqué est le prix à payer pour obtenir construire ce langage commun indispensable à ce surcroît de créativité potentielle.
} 
Ancori B., 2014. Communication, cognition et créativité dans les sociétés de la connaissance. À propos de deux dilemmes, Nouvelles perspectives en sciences sociales. Revue internationale d'analyse complexe et d'études relationnelles, 9 , 2, 45-93.

Ancori B., 2017. Complexité et créativité : émergence, stabilité et dynamiques des collectifs, Nouvelles perspectives en Sciences sociales. Revue Internationale de systémique complexe et d'études relationnelles, 12, 2, 11-39.

Axelrod R., Cohen M., 1999. Harnessing complexity. Organizational implications of a scientific frontier, New York, The Free Press. Trad. fr. : Réussir dans un monde complexe, Paris, Odile Jacob, 2001.

Bacqué M.-H., Sintomer Y. (Eds.), 2011. La démocratie participative. Histoire et généalogie, Paris, La Découverte, 2011.

Berlingen F., 2015. Face aux défis environnementaux et sociaux, des solutions communes en pair-à-pair, in Filippova D. (Ed.), Société collaborative. La fin des hiérarchies, Paris, Éditions Rue de l'Echiquier, 90-106.

Bonneuil C., Joly P.-B., 2013. Sciences, techniques et société, Paris, La Découverte.

Boyer R., Orléan A., 1994. Persistance et changement des conventions. Deux modèles simples et quelques illustrations, in Orléan A. (Ed.), Analyse économique des conventions, Paris, Presses Universitaires de France, 219-247.

Calame M., 2011. Lettre ouverte aux scientistes. Alternatives démocratiques à une idéologie cléricale, Paris, éditions Charles Léopold Mayer.

Callon M., Lascoumes P., Barthe Y., 2001. Agir dans un monde incertain. Essai sur la démocratie technique, Paris, Le Seuil.

Coutellec L., 2015. La science au pluriel. Essai d'épistémologie pour des sciences impliquées, Versailles, Quae.

David P., 1985. Clio and the economics of QWERTY, American Economic Review, 75, 2.

Gell-Mann M., 1995. Le quark et le jaguar. Voyage au coeur du simple et du complexe, Paris, Albin Michel.

Gibbons M., Limoges C., Nowotny H., Schwartzman S., Scott P., Trow M., 1994. The new production of knowledge: the dynamics of science and research in contemporary societies, London, Sage Publications.

Hess G., Bourg D. (Eds.), 2016. Science, conscience et environnement. Penser le monde complexe, Paris, Presses Universitaires de France.

Holland J.H., 1975. Adaptation in natural and artificial systems: an introductory analysis with applications to biology, Cambridge, MIT Press.

Houllier F. (Ed.), 2016. Les sciences participatives en France. État des lieux, bonnes pratiques \& recommandations, rapport au ministre de l'Éducation nationale, de l'Enseignement supérieur et de la Recherche, https://inradam-front-resources-cdn.brainsonic.com/ressources/afile/ 320323-7bb62-resource-rapport-de-la-mission-sciencesparticipatives-fevrier-2016.html.

Jollivet M., 2015. Pour une transition écologique citoyenne, Paris, Éditions Charles Léopold Mayer, 2015.
Larivière V., Archambault E., Gingras Y., 2008. Long-Term variations in the aging of scientific literature: from exponential growth to staedy-state science (1900-2004), Journal of the American Society for Information Science and Technology, 59, 2, 288-296.

Lascoumes P., 2012. Action publique et environnement, Paris, Presses Universitaires de France.

Laval C., Dardot P., 2015. Le principe du commun, principe d'un nouveau droit d'usage, Les Possibles, 5.

Laville B., 2010. Du ministère de l'impossible au ministère d'État, Revue française d'administration publique, 2, 134, 277-311.

Le Crosnier H., 2012. Elinor Ostrom ou la révolution des communs, Le Monde Diplomatique, 15 mai 2012.

Lewis D., 1969. Conventions: a philosophical study, Cambridge (MA), Harvard University Press.

Lewis D., 1983. Languages and Language, Philosophical Papers, Oxford University Press, Vol. 1.

Lipinsky M., 2011. Les sciences, un enjeu citoyen. Une politique écologiste de la recherche et de l'innovation, Paris, Les petits matins, 2011.

March J., 1991. Exploration and Exploitation in Organizational Learning, Organization Science, 2, 1, 71-87.

Meadows D.H, Meadows D.L., Randers J., Behrens III W.W., 1972. The Limits of Growth: a report for the Club of Rome's project on the predicament of mankind, New York, Universe Books.

Micoud A., 2001. La nébuleuse associative, in Boyer M., Herzlich G., Maresca B. (Eds.), L'environnement, question sociale. Dix ans de recherches pour le ministère de l'Environnement, Paris, Odile Jacob, 119-129.

Micoud A., 2007. De l'expert-militant à l'être vivant sensible», Cosmopolitiques, 15, juin, 121-134.

Nowotny H., Gibbons M., Scott P., 2001. Re-thinking science: knowledge production in the age of uncertainty, Oxford, Polity Press.

Ostrom E., 1990. Governing the commons: the evolution of institutions for collective action, Cambridge, Cambridge University Press.

Pestre D., 2003. Science, argent et politique. Un essai d'interprétation, Paris, INRA Éditions.

Pestre D., 2006. Introduction aux Science Studies, Paris, La Découverte.

Pestre D., 2008. La gouvernance des sciences en société, la gouvernance des sociétés en science : les tensions entre savoirs, pouvoirs et démocratie, in Alix J.-P., Ancori B., Petit P. (Eds.), Sciences en société au XXI siècle: autres relations, autres pratiques, Paris, CNRS Éditions, 41-56.

Pestre D., 2010. Des sciences et des productions techniques depuis trente ans. Chronique d'une mutation, Le Débat, 160, mai-août, 115-131.

Pestre D., 2011. Les sciences entre démiurgie, états de faits économiques et démocratie. Aperçu historique, situation présente, principes normatifs, in Bacqué M.-H., Sintomer Y. (Eds.), La démocratie participative. Histoire et généalogie, Paris, La Découverte, 261-282. 
Pestre D., 2013. À contre-science. Politiques et savoirs des sociétés contemporaines, Paris, Seuil, 2013.

Simon H., 1962. The architecture of complexity, Proceedings of the American Philosophy Society, 106, 467-482. Reproduit dans Simon H., Sciences des Systèmes, Sciences de l'artificiel, Paris, Bordas, 1991, 171-203.
Spanou C., 1991. Fonctionnaires et militants. L'administration et les nouveaux mouvements sociaux, Paris, L'Harmattan.

Stengers I., 2013. Une autre science est possible! Manifeste pour un ralentissement des sciences, suivi de James W., 1901, Le poulpe du doctorat, présenté par Drumm T., Paris, La Découverte.

Citation de l'article : Ancori B., 2017. Politiques de la recherche environnementale et complexité du social. Nat. Sci. Soc. 25, S76-S83. 